\title{
On another view of aggregation of fuzzy relations
}

\author{
Olga Grigorenko ${ }^{1}$ Julija Lebedinska ${ }^{2}$ \\ ${ }^{1}$ University of Latvia, Latvia \\ ${ }^{2}$ University of Latvia, Latvia
}

\begin{abstract}
The paper is devoted to a concept of aggregation of binary fuzzy relations. Consider two $n$-ary vectors, when we know the degrees to which corresponding components are in relation we can measure the degree to which vectors are in relation. Tasks with such background justify the necessity of aggregation of binary fuzzy relations and we contribute to the topic in this paper. First we recall the approach previously appeared in literature and then we introduce other approaches suitable for various practical applications. Later we study the property of $T$-transitivity since it indicates in some sense the consistency of aggregation.
\end{abstract}

Keywords: fuzzy relation, aggregation function, $T$ transitivity.

\section{Introduction}

Aggregation of fuzzy relations is important in fuzzy preference modeling (see e.g. [6]), decision making and solving other problems related to imprecise information (see e.g. [5])). Assume we have two vectors $\mathbf{x}=\left(x_{1}, \ldots, x_{n}\right)$ and $\mathbf{y}=\left(y_{1}, \ldots, y_{n}\right)$, we know the degree to which each particular pair of elements $\left(x_{i}, y_{i}\right), i \in\{1, \ldots, n\}$ is related, and thus we would like to know the degree to which vectors $\mathbf{x}$ and $\mathbf{y}$ are related. Aggregation of corresponding binary fuzzy relations is aimed at solution of this problem.

Method of pointwise aggregation of fuzzy relations is studied e.g. in [12]. We would like to contribute to the topic by proposing and studying other methods.

The paper is organized as follows: Section 2 following the introduction contains some basic notions and results necessary for better understanding of our research; we introduce notions of $\sigma$-aggregation and $A$ - $T$-aggregation of fuzzy relations in Section 3 , we also provide practical examples and motivations standing behind these formalizations; consistency of proposed aggregation methods is verified in Section 4, where we study the preservation of $T$-transitivity during the aggregation process; and we conclude the paper in Section 5 by outlining possible directions of the future researches.

\section{Preliminaries}

In the sequel we use the basic notions and properties of t-norms. For detailed information on t-norms the reader can refer e.g. to [9].

We recall the definition of aggregation function:

Definition 1. [7] An aggregation function is a mapping $A:[0,1]^{n} \rightarrow[0,1]$ which fulfills the following properties:

- $A\left(x_{1}, \ldots, x_{n}\right) \leq A\left(y_{1}, \ldots, y_{n}\right)$ whenever $x_{i} \leq y_{i}$ for all $i \in\{1, \ldots, n\}$ (monotonicity);

- $A(0, \ldots, 0)=0$ and $A(1, \ldots, 1)=1$ (boundary conditions).

For the details on aggregation functions the reader can see e.g. [7].

Although we employ the notion of aggregation function in our paper obtained results can be also extended to the aggregation operators (for details on aggregation operators refer to [10]).

We continue with an overview of basic definitions and results on fuzzy relations. First time definitions of fuzzy order relation and fuzzy equivalence relation were introduced by L.A. Zadeh in 1971 ([13]) under the names of fuzzy ordering and similarity relation. Fifteen years later U. Höhle and N. Blanchard in their paper [8] proposed to involve fuzzy equivalence relation (L-valued equality) into the definition of fuzzy order (partial ordering). In our paper we use more recent results on fuzzy order defined with respect to the fuzzy equivalence relation studied in [2].

Definition 2. A fuzzy binary relation $R$ on a set $X$ is a mapping $R: X \times X \rightarrow[0,1]$.

Definition 3. (see e.g. [13], [1]) A fuzzy binary relation $E$ on a set $X$ is called fuzzy equivalence relation (or T-equivalence) with respect to a $t$-norm $T$, if and only if the following three axioms are fulfilled for all $x, y, z \in X$ :

1) $E(x, x)=1$ reflexivity;

2) $E(x, y)=E(y, x)$ symmetry;

3) $T(E(x, y), E(y, z)) \leq E(x, z) T$-transitivity.

The following result establishes principles of construction of fuzzy equivalence relations using pseudo-metrics.

Theorem 1. (see e.g.[1], [2]) Let T be a continuous Archimedean t-norm with an additive generator $t$. 
For any pseudo-metric $d$, the mapping $E_{d}(x, y)=$ $t^{(-1)}(\min (d(x, y), t(0)))$ is a $T$-equivalence.

Example 1. Let us consider the set of real numbers $X=\mathbb{R}$ and metric $d(x, y)=|x-y|$ on it. Taking into account that $t_{L}(x)=1-x$ is an additive generator of $T_{L}$ (Lukasievicz $t$-norm) and that $t_{P}(x)=-\ln (x)$ is an additive generator of $T_{P}$ (product t-norm), we obtain two fuzzy equivalence relations:

$$
E_{L}(x, y)=\max (1-|x-y|, 0) ;
$$

Definition 4. [2] Let $\preceq$ be a crisp order on $X$ and let $E$ be a fuzzy equivalence relation on $X . E$ is called compatible with $\preceq$ if and only if the following implication holds for all $x, y, z \in X: x \preceq y \preceq z \Rightarrow$ $(E(x, z) \leq E(y, z)$ and $E(x, z) \leq E(x, y))$.

Remark 1. Let $X$ be the set of real numbers and $\leq$ be a linear order on it. Then, for a fixed element $x_{0}$, $E\left(x, x_{0}\right)$ is non-decreasing in the interval $\left[-\infty, x_{0}\right]$ and non-increasing in the interval $\left[x_{0}, \infty\right]$, where $E$ is a fuzzy equivalence relation which is compatible with $\leq$.

Definition 5. (see e.g. [2]) A T-transitive fuzzy relation $L: X \times X \rightarrow[0,1]$ is called fuzzy order relation with respect to a t-norm $T$ and $a$ $T$-equivalence $E$ (or T-E-order), if and only if it additionally fulfills the following two axioms for all $x, y \in X:$

1) $L(x, y) \geq E(x, y)$ E-reflexivity;

2) $T(L(x, y), L(y, x)) \leq E(x, y) T$-E-antisymmetry.

A fuzzy order $L$ is called strongly linear if and only if $\forall x, y \in X: \max (L(x, y), L(y, x))=1$.

The following theorem states that strongly linear fuzzy order relations are uniquely characterized as fuzzifications of crisp linear orders.

Theorem 2. [2] Let $L$ be a binary fuzzy relation on $X$ and let $E$ be a T-equivalence on $X$. Then the following two statements are equivalent:

1. $L$ is a strongly linear $T$-E-order on $X$.

2. There exists a linear order $\preceq$ the relation $E$ is compatible with, such that $L$ can be represented as follows:

$$
L(x, y)=\left\{\begin{array}{lc}
1, & \text { if } x \preceq y \\
E(x, y), & \text { otherwise } .
\end{array}\right.
$$

This theorem shows that if we have a set $X$, a linear order $\preceq$ on it and a $T$-equivalence on $X$ which is compatible with $\preceq$, then we can build a fuzzy linear order as it was shown above.

Further, in some results we consider special type of fuzzy order relation on interval $[0,1]$ :

$$
L(x, y)=\left\{\begin{array}{lc}
1, & \text { if } x \leq y \\
g(|x-y|), & \text { otherwise }
\end{array}\right.
$$

where $g$ is a non-increasing function.

For more information regarding to fuzzy orders constructed on the base of fuzzy equivalence relations see [5],[4].

In general $L$ defined by (1) is not a fuzzy order relation. The choice of a function $g$ is important. The necessary condition for the relation $E(x, y)=$ $g(|x-y|)$ to be compatible with $\leq$ is that function $g$ is non-increasing. Therefore, if we prove a result for an arbitrary fuzzy relation $L$ defined as above, the result will also hold for a fuzzy order relation

$$
R(x, y)= \begin{cases}1, & \text { if } x \leq y \\ E(x, y)=g(|x-y|), & \text { otherwise }\end{cases}
$$

where $E$ is a fuzzy equivalence relation compatible with $\leq$.

Fuzzy order relation defined as above can be widely used in practical applications, see e.g. [5].

In our paper, studying the process of $A-T$ aggregation, we focus on the preservation of $T$ transitivity where initial fuzzy order relation are given by (2).

\section{Motivation and construction}

In this section first we recall the notion of pointwise aggregation of binary fuzzy relations studied in [12]. Later we define aggregation of binary fuzzy relations by means of a t-norm $T$ and an arbitrary (or the particular) aggregation function $A$. We propose two methods aimed at the aggregation of $n$ binary fuzzy relations. We call the first one $\sigma$-aggregation and it is similar to the definition of pointwise aggregation with one more assumption: it allows arbitrary permutations of arguments. The second one we call $A$ - $T$-aggregation, it employs ideas of the extension principle (see e.g. [14], [11]) and it conceptually differs from approaches described before.

For the sake of brevity further we denote vectors in bold e.g. $\mathbf{x}=\left(x_{1}, \ldots, x_{n}\right), \mathbf{y}^{*}=\left(y_{1}^{*}, \ldots, y_{n}^{*}\right)$.

Definition 6. $[12]^{1}$ Let $R_{i}: X_{i} \times X_{i} \rightarrow[0,1]$, $i \in\{1, \ldots, n\}$ be binary fuzzy relations, $A:[0,1]^{n} \rightarrow[0,1]$ be an arbitrary aggregation function then

$$
\tilde{R}(\mathbf{x}, \mathbf{y})=A\left(R_{1}\left(x_{1}, y_{1}\right), \ldots, R_{n}\left(x_{n}, y_{n}\right)\right)
$$

is called pointwise aggregation of fuzzy relations.

$$
\tilde{R}: X \times X \rightarrow[0,1] \text {, where } X=\prod_{i} X_{i} \text {, given by }
$$

Definition 6 allows us to define the value to which arbitrary vectors $\mathbf{x}, \mathbf{y}$ are in relation.

\footnotetext{
${ }^{1}$ the original definition is given for the aggregation operator or extended aggregation function, in order to align the compared approaches we consider the case of aggregation function, i.e. when the number of augments is fixed. Furthermore in the original paper this process is called aggregation of fuzzy relations, but we call it pointwise aggregation to distinguish this approach from other approaches introduced in the paper.
} 
As it is described in [12], aggregation of fuzzy relations can be used in data querying problems. If we assume that $\mathbf{x}=\left(x_{1}, \ldots, x_{n}\right)$ is a query, where $x_{i}$ is a value referring to the $i$-th field of the query, and $\mathbf{y}=\left(y_{1}, \ldots, y_{n}\right)$ is an arbitrary data record, then $\tilde{R}$ given by Definition 6 measures the degree to which $\mathbf{y}$ matches query $\mathbf{x}$.

Let us now introduce the notion of $\sigma$-aggregation:

Definition 7. Let $R_{i}: X_{i} \times X_{i} \rightarrow[0,1]$, $i \in\{1, \ldots, n\}$ be binary fuzzy relations,

$A$ be an arbitrary aggregation function and $\left(y_{\sigma(1)}, \ldots, y_{\sigma(n)}\right)$ be an arbitrary permutation of vector $\mathbf{y}\left(\sigma\right.$ is a permutation from the set $S_{n}$ of all permutations of the set $\{1, \ldots, n\})$. Then

$\tilde{R}_{\sigma}(\mathbf{x}, \mathbf{y})=\max _{\sigma \in S_{n}}\left\{A\left(R_{1}\left(x_{1}, y_{\sigma(1)}\right), \ldots, R_{n}\left(x_{n}, y_{\sigma(n)}\right)\right)\right\}$

is called $\sigma$-aggregation.

The domain and co-domain of $\tilde{R}_{\sigma}$ are the same like in the case of $\tilde{R}$ :

$\tilde{R}_{\sigma}: X \times X \rightarrow[0,1]$, where $X=\prod_{i} X_{i}$

The necessity of $\tilde{R}_{\sigma}$ is justified by similar practical needs as in the case of pointwise aggregation.

Let's consider the example:

Example 2. $\tilde{R}_{\sigma}$ can measure the degree to which a query $\mathbf{y}$ matches a query $\mathbf{x}$, where all records are sentences and all elements of the record are words in the language where the order of words is not of the vital importance, e.g. Slavonic, Baltic languages or English in some particular cases.

In this case we should use only one fuzzy relation $R$ (not different $R_{i}$ for each attribute $i$ ), because symmetry need to be preserved. Since we allow the arbitrary permutations of the elements of vectors preservation of symmetry is not possible when we have different $R_{i}$. Moreover to preserve the property of symmetry we should introduce a symmetric aggregation function.

Let consider the query "When I am in Latvia I usually visit Jurmala", if we apply $\tilde{R}_{\sigma}$ it is very well matched to the query "I usually visit Jurmala when I am in Latvia", since it disregards the order of words. We can expect $\tilde{R}_{\sigma}$ value close to 1 and this is good reflection of the reality since both queries contain the same information. In this case the classical pointwise aggregation does not work.

Now we define $A$ - $T$-aggregation and justify its necessity.

Definition 8. Let $R_{i}:[0,1] \times[0,1] \rightarrow[0,1]$,

$i \in\{1, \ldots, n\}$ be binary fuzzy relations,

$A:[0,1]^{n} \rightarrow[0,1]$ be an arbitrary aggregation func-

tion, $T$ be an arbitrary $t$-norm then

$\tilde{R}_{A, T}:[0,1] \times[0,1] \rightarrow[0,1]$ given by

$\tilde{R}_{A, T}(x, y)=\sup _{\substack{\left(x_{1}, \ldots, x_{n}\right),\left(y_{1}, \ldots, y_{n}\right)}}\left\{T\left(R_{1}\left(x_{1}, y_{1}\right), \ldots, R_{n}\left(x_{n}, y_{n}\right)\right):\right.$

$\left.A\left(x_{1}, \ldots, x_{n}\right)=x, A\left(y_{1}, \ldots, y_{n}\right)=y\right\}$

is called $A$-T-aggregation of fuzzy relations.
We require the domain of $R_{i}$ to be $[0,1] \times[0,1]$, because we aggregate elements from the corresponding set and we consider aggregation functions defined on $[0,1]^{n}$. Although extension to aggregation functions defined on $\mathbb{I}^{n}$, where $\mathbb{I}$ is a nonempty real interval, is a matter of rescaling and with few changes it can be easily done. In this case the domain of the relation $\tilde{R}_{A, T}$ would be $\mathbb{I} \times \mathbb{I}$.

The essence of $A$-T-aggregation is similar as in previous cases: we compare two $n$-ary vectors by fusing corresponding binary fuzzy relations. However in this case we take into account the aggregation of corresponding vectors.

Remark 2. In our work we will use the above definition of A-T-aggregation of fuzzy relations, but we present here also an alternative definition.

Let $R_{i}:[0,1] \times[0,1] \rightarrow[0,1]$,

$i \in\{1, \ldots, n\}$ be binary fuzzy relations,

$A:[0,1]^{n} \rightarrow[0,1]$ be an arbitrary aggregation function, $T$ be an arbitrary $t$-norm. Then

$$
\begin{gathered}
\tilde{R}_{A, T}(\mathbf{x}, \mathbf{y})=\sup _{\substack{\left(x_{1}^{\prime}, \ldots, x_{n}^{\prime}\right),\left(y_{1}^{\prime}, \ldots, y_{n}^{\prime}\right)}}\left\{T\left(R_{1}\left(x_{1}^{\prime}, y_{1}^{\prime}\right), \ldots, R_{n}\left(x_{n}^{\prime}, y_{n}^{\prime}\right)\right):\right. \\
A\left(x_{1}^{\prime}, \ldots, x_{n}^{\prime}\right)=A\left(x_{1}, \ldots, x_{n}\right) \\
\left.A\left(y_{1}^{\prime}, \ldots, y_{n}^{\prime}\right)=A\left(y_{1}, \ldots, y_{n}\right)\right\}
\end{gathered}
$$

is called T-A-aggregation of fuzzy relations.

The domain and co-domain of $\tilde{R}_{T, A}$ in this case are the same like in the case of $\tilde{R}$ :

$$
\tilde{R}_{A, T}: X \times X \rightarrow[0,1],
$$

where $X=[0,1]^{n}$.

This definition shows similarity of approaches.

And now let us justify the concept of $A-T$ aggregation.

The definition of monotonicity for an aggregation function $A$ says that if $x_{i} \leq y_{i}$ for all $i \in\{1, \ldots, n\}$ then $A\left(x_{1}, \ldots, x_{n}\right) \leq A\left(y_{1}, \ldots, y_{n}\right)$. Then for results of aggregation $x$ and $y$ we definitely know that $x \leq y$ whenever there exist vectors $\left(x_{1}, \ldots, x_{n}\right)$ and $\left(y_{1}, \ldots, y_{n}\right)$ such that $A\left(x_{1}, \ldots, x_{n}\right)=x$ and $A\left(y_{1}, \ldots, y_{n}\right)=y$ and $x_{i} \leq y_{i}$ for all $i \in\{1, \ldots, n\}$.

Now let imagine that for each coordinate we have different order relations:

$$
x_{1} \leq_{1} y_{1}, \ldots, x_{n} \leq_{n} y_{n} .
$$

Then if we continue by using fuzzy language we can rewrite:

$$
x \leq y \text { as } \tilde{R}(x, y)
$$

and

$$
x_{i} \leq_{i} y_{i} \text { as } R_{i}\left(x_{i}, y_{i}\right) \text { for all } i \in\{1, \ldots, n\} .
$$

As a connective we use a t-norm $T$, and, finally, instead of "for all" we use supremum. Thus we get

$$
\tilde{R}(x, y)=\sup _{\substack{\left(x_{1}, \ldots, x_{n}\right),\left(y_{1}, \ldots, y_{n}\right)}}\left\{T\left(R_{1}\left(x_{1}, y_{1}\right), \ldots, R_{n}\left(x_{n}, y_{n}\right)\right):\right.
$$




$$
\left.A\left(x_{1}, \ldots, x_{n}\right)=x, A\left(y_{1}, \ldots, y_{n}\right)=y\right\},
$$

what is exactly the Definition 8. By the example of monotonicity property we have shown that property of aggregated results is in relation with the property of elements of vectors, which aggregate to these results, where the relations's strength is defined by t-norm $T$. In this example we spoke about order relations but the same reasoning works also for equivalence relations.

The alternative motivation could be as follows:

We know that if $x_{1} \leq y_{1}, \ldots, x_{n} \leq y_{n}$ then $x \leq y$ (where $A\left(x_{1}, \ldots, x_{1}\right)=x$ and $A\left(y_{1}, \ldots, y_{1}\right)=y$ ).

Using fuzzy order relations and a t-norm $T$ as a connective we could rewrite:

$$
T\left(R_{1}\left(x_{1}, y_{1}\right), \ldots, R_{n}\left(x_{n}, y_{n}\right)\right) \leq \tilde{R}(x, y) .
$$

Since we could rewrite the above inequality for all vectors which are aggregated to $x$ and $y$, it is natural to define $\tilde{R}(x, y)$ as in the Definition 8 .

To illustrate the essence of $A-T$-aggregation let us give an example.

Example 3. We consider a data set describing progress in studies of students. For each student the estimations in tree main courses are given in a table. The data set is shown in Table 1:

Table 1: Evaluation of students

\begin{tabular}{|l|l|l|l|l|}
\hline Students & $\begin{array}{l}\text { Course } \\
1\end{array}$ & $\begin{array}{l}\text { Course } \\
2\end{array}$ & $\begin{array}{l}\text { Course } \\
2\end{array}$ & $\begin{array}{l}\text { Aggreg. } \\
\text { result }\end{array}$ \\
\hline$s_{1}$ & 0.8 & 0.7 & 0.6 & 0.7 \\
\hline$s_{2}$ & 0.8 & 1 & 1 & 0.93 \\
\hline$s_{3}$ & 0.7 & 0.9 & 1 & 0.87 \\
\hline$s_{4}$ & 0.6 & 0.8 & 0.9 & 0.77 \\
\hline$s_{5}$ & 0.8 & 0.9 & 0.6 & 0.77 \\
\hline$s_{6}$ & 0.6 & 0.7 & 0.5 & 0.6 \\
\hline$s_{7}$ & 0.8 & 0.5 & 1 & 0.77 \\
\hline$s_{8}$ & 0.9 & 0.5 & 0.8 & 0.73 \\
\hline$s_{9}$ & 0.7 & 0.9 & 0.6 & 0.73 \\
\hline$s_{10}$ & 0.7 & 0.8 & 0.8 & 0.77 \\
\hline
\end{tabular}

In this table the students' results are given as well as the aggregated values of the results in three different courses, the arithmetic mean is used to aggregate the results. And let now imagine that we have to compare all the students. We not only want to know for a pair of students $s_{i}$ and $s_{j}$ which student is better, but we want to know the degree to which student $s_{i}$ is better than student $s_{j}$. Let us introduce the fuzzy order relations $R_{i}$ to compare the marks of students in course $i$ by the following way:

$$
R_{i}\left(a_{i}, b_{i}\right)=\left\{\begin{array}{lc}
1, & \text { if } a_{i} \leq b_{i} \\
\max \left(1-\left|a_{i}-b_{i}\right|, 0\right), & \text { otherwise }
\end{array} .\right.
$$

In this example we introduce the same fuzzy order relations for all coordinates $i \in\{1,2,3\}$, but in general they can be different. Let us compare the students $s_{9}$ and $s_{10}$ or, namely, let calculate the degree to which the student $s_{9}$ is worse than student $s_{10}$ (or, in other words, the degree to which student $s_{10}$ is better than student $\left.s_{9}\right)$. If we use pointwise aggregation and minimum as an aggregation function, the result will be $\tilde{R}\left(s_{9}, s_{10}\right)=0.9$.

However if we want to compare the results of students, taking into account aggregated results (and disregarding in some sense each course result), we should use the A-T-aggregation (A-arithmetic mean). If we use minimum t-norm, then the result will be:

$\tilde{R}_{A, T}\left(s_{9}, s_{10}\right)=1$ since there exists a vector $(0.8,0.9,0.6)$ such that $A(0.8,0.9,0.6)=A(0.7,0.8,0.8)=0.77$ and $\min \left(R_{1}(0.7,0.8), R_{2}(0.9,0.9), R_{3}(0.6,0.6)\right)=1$.

$A$-T-aggregation is useful in the cases when aggregated results are much more important than each component itself.

\section{T-transitivity}

This section is devoted to the preservation of $T$ transitivity property during the aggregation processes described before. We use notation $T^{*}$ for the t-norm used in transitivity property to distinguish from the t-norm used in the aggregation and correspondingly we write $T^{*}$-transitivity. Preservation of $T^{*}$-transitivity in some sense indicates how consistent is our aggregation, therefore it is of our particular interest. We remind the definition of $T^{*}$ transitivity for fuzzy relations:

Definition 9. (see e.g. [8]) Consider a binary fuzzy relation $R$ on a set $X$ and an arbitrary t-norm $T^{*}$. $R$ is called $T^{*}$-transitive if for all $x, y, z \in X$ the following property holds:

$$
T^{*}(R(x, y), R(y, z)) \leq R(x, z) .
$$

In the same manner $T^{*}$-transitivity for aggregated fuzzy relation is defined:

Definition 10. $\tilde{R}$ is called $T^{*}$-transitive if for all corresponding $\mathbf{x}, \mathbf{y}, \mathbf{z}$ the following property holds:

$$
T^{*}(\tilde{R}(\mathbf{x}, \mathbf{y}), \tilde{R}(\mathbf{y}, \mathbf{z})) \leq \tilde{R}(\mathbf{x}, \mathbf{z}) .
$$

We define $T^{*}$-transitivity of $\tilde{R}_{\sigma}, \tilde{R}_{A, T}$ in the same way like in Definition 10.

\subsection{Pointwise aggregation}

Notion of aggregation functions dominance is crucial for preservation of $T^{*}$-transitivity of $\tilde{R}$ :

Definition 11. [12] Consider an n-argument aggregation function $A^{n}:[0,1]^{n} \rightarrow[0,1]$ and an $m$ argument aggregation function $B^{m}:[0,1]^{m} \rightarrow[0,1]$. We say that $A^{n}$ dominates $B^{m}$ if for all $x_{i, j} \in[0,1]$ 
with $i \in\{1, \ldots, m\}$ and $j \in\{1, \ldots, n\}$ the following property holds:

$$
\begin{aligned}
& B^{m}\left(A^{n}\left(x_{1,1}, \ldots, x_{1, n}\right), \ldots, A^{n}\left(x_{m, 1}, \ldots, x_{m, n}\right)\right) \leq \\
& \leq A^{n}\left(B^{m}\left(x_{1,1}, \ldots, x_{m, 1}\right), \ldots, B^{m}\left(x_{1, n}, \ldots, x_{m, n}\right)\right) .
\end{aligned}
$$

The following result for the pointwise aggregation can be found in [12].

Theorem 3. [12] Let $|X|>3$ and let $T^{*}$ be a t-norm. An aggregation function $A$ preserves $T^{*}$ transitivity of fuzzy relations on $X$ if and only if $A$ belongs to the class of aggregation functions which dominate $T^{*}$.

\section{2. $\sigma$-aggregation}

In this subsection we outline the properties necessary for the preservation of $T^{*}$-transitivity in the case of $\sigma$-aggregation.

Theorem 4. If $R$ is a $T^{*}$-transitive fuzzy relation, and $A$ is a symmetric aggregation function which dominates $T^{*}$ then $\tilde{R}_{\sigma}$ is also $T^{*}$-transitive, where

$$
\tilde{R}_{\sigma}(\mathbf{x}, \mathbf{y})=\max _{\sigma \in S_{n}}\left\{A\left(R\left(x_{1}, y_{\sigma(1)}\right), \ldots, R\left(x_{n}, y_{\sigma(n)}\right)\right)\right\}
$$

Proof. We need to show that

$$
T^{*}\left(\tilde{R}_{\sigma}(\mathbf{x}, \mathbf{y}), \tilde{R}_{\sigma}(\mathbf{y}, \mathbf{z})\right) \leq \tilde{R}_{\sigma}(\mathbf{x}, \mathbf{z})
$$

for arbitrary $\mathbf{x}, \mathbf{y}, \mathbf{z}$.

One can see that if we use symmetric aggregation function and only one fuzzy relation $R$ for all coordinates $i \in\{1, \ldots, n\}$, it is not important which one of the vectors $\mathbf{x}$ or $\mathbf{y}$ or both of them we permute in Definition 7 , result is the same. Therefore by Definition 7 there exists $\mathbf{x}^{*}=\left(x_{1}^{*}, \ldots, x_{n}^{*}\right)$ the permutation of $\mathbf{x}$ such that

$$
\tilde{R}_{\sigma}(\mathbf{x}, \mathbf{y})=A\left(R\left(x_{1}^{*}, y_{1}\right), \ldots, R\left(x_{n}^{*}, y_{n}\right)\right) .
$$

Similarly there exists $\mathbf{z}^{*}=\left(z_{1}^{*}, \ldots, z_{n}^{*}\right)$ the permutation of $\mathbf{z}$ such that

$$
\tilde{R}_{\sigma}(\mathbf{y}, \mathbf{z})=A\left(R\left(y_{1}, z_{1}^{*}\right), \ldots, R\left(y_{n}, z_{n}^{*}\right)\right)
$$

By the fact that $A$ dominates $T^{*}$ and that $R$ is the $T^{*}$-transitive fuzzy relation we conclude:

$$
\begin{gathered}
T^{*}\left(\tilde{R}_{\sigma}(\mathbf{x}, \mathbf{y}), \tilde{R}_{\sigma}(\mathbf{y}, \mathbf{z})\right)= \\
T^{*}\left(A\left(R\left(x_{1}^{*}, y_{1}\right), \ldots, R\left(x_{n}^{*}, y_{n}\right)\right),\right. \\
\left.A\left(R\left(y_{1}, z_{1}^{*}\right), \ldots, R\left(y_{n}, z_{n}^{*}\right)\right)\right) \leq \\
\leq A\left(T^{*}\left(R\left(x_{1}^{*}, y_{1}\right), R\left(y_{1}, z_{1}^{*}\right)\right), \ldots,\right. \\
\left.\ldots, T^{*}\left(R\left(x_{n}^{*}, y_{n}\right), R\left(y_{n}, z_{n}^{*}\right)\right)\right) \leq \\
\leq A\left(R\left(x_{1}^{*}, z_{1}^{*}\right), \ldots, R\left(x_{n}^{*}, z_{n}^{*}\right)\right) \leq \tilde{R}_{\sigma}(\mathbf{x}, \mathbf{z}) .
\end{gathered}
$$

\section{3. $A$-T-aggregation}

In this section we consider special type of fuzzy order relations given by formula 2 and explore when $A$ - $T$-aggregation of such fuzzy order relations preserves $T^{*}$-transitivity.

We denote $A_{x}$ the set of vectors $\mathbf{x}$ such as $A(\mathbf{x})=x$, similarly we denote $A_{y}$.

In the next two theorems we show that in some cases in order to calculate the value $\tilde{R}_{A, T}(x, y)$ it is sufficient to know the values $R_{i}(x, y)$ for all $i$.

Theorem 5. If $x \leq y, A$ is an arbitrary idempotent aggregation function, $R_{i}, i \in\{1, \ldots, n\}$ are given by formula 2 then $\tilde{R}_{A, T}(x, y)=1$.

Proof. By idempotence of $A$ we have $A(x, \ldots, x)=x$ and $A(y, \ldots, y)=y$. Since $x \leq y$ we conclude that $(x, \ldots, x) \leq(y, \ldots, y)$ componentwise.

Since $x \leq y, R_{i}(x, y)=1$ and hence

$$
\tilde{R}_{A, T}(x, y) \geq T\left(R_{1}(x, y), \ldots, R_{n}(x, y)\right)=1 .
$$

Theorem 6. If $R_{i}$ for all $i \in\{1, \ldots, n\}$ are fuzzy relations, $T=T_{M}$ (minimum t-norm), $A$ is an idempotent aggregation function, satisfying the following condition for all $\left(x_{1}, \ldots, x_{n}\right) \in A_{x},\left(y_{1}, \ldots, y_{n}\right) \in A_{y}$ and for all $i \in\{1, \ldots, n\}$ :

$R_{i}(x, y) \geq T_{M}\left(R_{i}\left(x_{1}, y_{1}\right), R_{i}\left(x_{2}, y_{2}\right), \ldots, R_{i}\left(x_{n}, y_{n}\right)\right)$

then

$$
\tilde{R}_{A, T}(x, y)=T_{M}\left(R_{1}(x, y), \ldots, R_{n}(x, y)\right) .
$$

Proof. Using

$R_{i}(x, y) \geq T_{M}\left(R_{i}\left(x_{1}, y_{1}\right), R_{i}\left(x_{2}, y_{2}\right), \ldots, R_{i}\left(x_{n}, y_{n}\right)\right)$

by idempotence of $T_{M}$ we get

$$
\begin{gathered}
T_{M}\left(R_{i}(x, y), \ldots, R_{i}(x, y)\right) \geq \\
\geq T_{M}\left(R_{i}\left(x_{1}, y_{1}\right), R_{i}\left(x_{2}, y_{2}\right), \ldots, R_{i}\left(x_{n}, y_{n}\right)\right)
\end{gathered}
$$

for all relations $R_{i}, i \in\{1, \ldots, n\}$.

Therefore, by monotonicity of $T_{M}$ we get $T_{M}\left(T_{M}\left(R_{1}(x, y), \ldots, R_{1}(x, y)\right), \ldots\right.$,

$$
\begin{gathered}
\left.\ldots, T_{M}\left(R_{n}(x, y), \ldots, R_{n}(x, y)\right)\right) \geq \\
\geq T_{M}\left(T_{M}\left(R_{1}\left(x_{1}, y_{1}\right), \ldots, R_{1}\left(x_{n}, y_{n}\right)\right), \ldots,\right. \\
\left.\ldots, T_{M}\left(R_{n}\left(x_{1}, y_{1}\right), \ldots, R_{n}\left(x_{n}, y_{n}\right)\right)\right) .
\end{gathered}
$$

Using associativity of $T_{M}$ we continue in the following way:

$$
\begin{aligned}
& T_{M}\left(T_{M}\left(R_{1}(x, y), \ldots, R_{n}(x, y)\right), \ldots,\right. \\
&\left.\ldots, T_{M}\left(R_{1}(x, y), \ldots, R_{n}(x, y)\right)\right) \geq \\
& \geq T_{M}\left(T_{M}\left(R_{1}\left(x_{1}, y_{1}\right), \ldots, R_{n}\left(x_{n}, y_{n}\right)\right), \ldots,\right. \\
&\left.\ldots, T_{M}\left(R_{1}\left(x_{1}, y_{1}\right), \ldots, R_{n}\left(x_{n}, y_{n}\right)\right)\right) .
\end{aligned}
$$

Using idempotence of $T_{M}$ from the previous we deduce that 


$$
\begin{aligned}
& T_{M}\left(R_{1}(x, y), \ldots, R_{n}(x, y)\right) \geq \\
\geq & T_{M}\left(R_{1}\left(x_{1}, y_{1}\right), \ldots, R_{n}\left(x_{n}, y_{n}\right)\right) .
\end{aligned}
$$

Since the previous inequality holds for arbitrary vectors $\left(x_{1}, \ldots, x_{n}\right) \in A_{x},\left(y_{1}, \ldots, y_{n}\right) \in A_{y}$ it also holds for the supremum:

$$
\begin{gathered}
\sup _{M} T_{M}\left(R_{1}(x, y), \ldots, R_{n}(x, y)\right) \geq \\
\left.\operatorname{su}_{M}\left(R_{1}\left(x_{1}, y_{1}\right), \ldots, R_{n}\left(x_{n}, y_{n}\right)\right)\right\}= \\
\left(y_{1}, \ldots, y_{n}\right) \in A_{y}, \\
=\tilde{R}_{A, T}(x, y)
\end{gathered}
$$

But $(x, \ldots, x) \in A_{x},(y, \ldots, y) \in A_{y}$ thus

$$
\tilde{R}_{A, T}(x, y)=T_{M}\left(R_{1}(x, y), \ldots, R_{n}(x, y)\right) .
$$

It is easy to see, that if $x>y$ and fuzzy relation $R_{i}$ is given by formula 2 then the property

$$
R_{i}(x, y) \geq T_{M}\left(R_{i}\left(x_{1}, y_{1}\right), R_{i}\left(x_{2}, y_{2}\right), \ldots, R_{i}\left(x_{n}, y_{n}\right)\right)
$$

is equivalent to the property that there exists an index $s$ such that

$$
x-y \leq x_{s}-y_{s}
$$

Now we show that $\tilde{R}_{A, T}$ based on additive aggregation function has the property described in the previous theorem.

Theorem 7. If $x>y, R_{i}$ for all $i \in\{1, \ldots, n\}$ are fuzzy relations given by formula 2, $T=T_{M}, A$ is an additive aggregation function then

$R_{i}(x, y) \geq T_{M}\left(R_{i}\left(x_{1}, y_{1}\right), R_{i}\left(x_{2}, y_{2}\right), \ldots, R_{i}\left(x_{n}, y_{n}\right)\right)$, for all $\left(x_{1}, \ldots, x_{n}\right) \in A_{x},\left(y_{1}, \ldots, y_{n}\right) \in A_{y}$ and for all $i \in\{1, \ldots, n\}$.

Proof. Let us consider an arbitrary $R_{i}$, $i \in\{1, \ldots, n\}$. We need to show for arbitrary $\left(x_{1}, \ldots, x_{n}\right) \in A_{x}$ and $\left(y_{1}, \ldots, y_{n}\right) \in A_{y}$ that $R_{i}(x, y) \geq T_{M}\left(R_{i}\left(x_{1}, y_{1}\right), R_{i}\left(x_{2}, y_{2}\right), \ldots, R_{i}\left(x_{n}, y_{n}\right)\right)=$ $=\min _{k \cdot x_{k}>y_{k}} R_{i}\left(x_{k}, y_{k}\right)$. The last equality holds since $R_{i}\left(x_{j}, y_{j}\right)=1$ if $x_{j} \leq y_{j}$.

Equally, because of the nature of the fuzzy order relation $R_{i}$ (see formula 2 ), it is sufficient to show that

$$
x-y \leq \max _{k: x_{k}>y_{k}}\left(x_{k}-y_{k}\right)
$$

We have: $x-y=A\left(x_{1}, \ldots, x_{n}\right)-A\left(y_{1}, \ldots, y_{n}\right)$. Now we substitute arguments of $A\left(x_{1}, \ldots, x_{n}\right)$ standing in the positions $l: x_{l} \leq y_{l}$ by the corresponding coordinate from the vector $\left(y_{1}, \ldots, y_{n}\right)$. So, by monotonicity of $A$ we estimate:

$$
\begin{gathered}
x-y=A\left(x_{1}, \ldots, x_{n}\right)-A\left(y_{1}, \ldots, y_{n}\right) \leq \\
A\left(x_{1}, \ldots, y_{l}, \ldots, x_{n}\right)-A\left(y_{1}, \ldots, y_{l}, \ldots, y_{n}\right) .
\end{gathered}
$$

Further by additivity of $A$ we get:

$$
x-y \leq A\left(x_{1}-y_{1}, \ldots, 0, \ldots, x_{n}-y_{n}\right) .
$$

Since additivity implies idempotence we have the required inequality:

$$
x-y \leq \max _{k: x_{k}>y_{k}}\left(x_{k}-y_{k}\right) .
$$

Thus we have shown that the condition holds.

From the previous two theorems we have the following result:

Conclusion 1. If $R_{i}$ for all $i \in\{1, \ldots, n\}$ are fuzzy relations given by formula $2, T=T_{M}, A$ is an additive aggregation function then $\tilde{R}_{A, T_{M}}$ could be represented as follows:

$$
\tilde{R}_{A, T_{M}}(x, y)=\left\{\begin{array}{l}
1, \text { if } x \leq y \\
T_{M}\left(R_{1}(x, y), \ldots, R_{n}(x, y)\right), \text { oth. }
\end{array}\right.
$$

Now we focus on preservation of $T^{*}$-transitivity:

Theorem 8. If $R_{i}$ for all $i \in\{1, \ldots, n\}$ are $T^{*}$-transitive fuzzy order relations given by formula 2:

$$
R_{i}(x, y)=\left\{\begin{array}{lc}
1, & \text { if } x \leq y \\
g_{i}(|x-y|), & \text { otherwise }
\end{array}\right.
$$

where $g_{i}$ are non-increasing mappings, $A$ is an aggregation function and aggregated fuzzy relation $\tilde{R}_{A, T}$ is represented as follows:

$$
\tilde{R}_{A, T}(x, y)=\left\{\begin{array}{l}
1, \text { if } x \leq y \\
T\left(R_{1}(x, y), \ldots, R_{n}(x, y)\right), \quad \text { oth } .
\end{array}\right.
$$

then $\tilde{R}_{A, T}$ is a $T^{*}$-transitive fuzzy order relation.

Proof. We have to prove that

$$
T^{*}\left(\tilde{R}_{A, T}(x, y), \tilde{R}_{A, T}(y, z)\right) \leq \tilde{R}_{A, T}(x, z) .
$$

Let us observe four different cases.

Consider $x \leq y$ and $y \leq z$, then $x \leq z$ and $\tilde{R}_{A, T}(x, z)=1$. And thus

$$
T^{*}\left(\tilde{R}_{A, T}(x, y), \tilde{R}_{A, T}(y, z)\right) \leq \tilde{R}_{A, T}(x, z) .
$$

Consider $x>y$ and $y \leq z$. If $x \leq z$ then the inequality $T^{*}\left(\tilde{R}_{A, T}(x, y), \tilde{R}_{A, T}(y, z)\right) \leq \tilde{R}_{A, T}(x, z)$ holds since $\tilde{R}_{A, T}(x, z)=1$. Therefore we consider the case when $x>z$. Thus $y \leq z<x$ and

$$
\begin{gathered}
T^{*}\left(\tilde{R}_{A, T}(x, y), \tilde{R}_{A, T}(y, z)\right)= \\
=\tilde{R}_{A, T}(x, y)=T_{M}\left(R_{1}(x, y), \ldots, R_{n}(x, y)\right)= \\
=T_{M}\left(g_{1}(|x-y|), g_{2}(|x-y|), \ldots, g_{n}(|x-y|)\right) \leq \\
\leq T_{M}\left(g_{1}(|x-z|), g_{2}(|x-z|), \ldots, g_{n}(|x-z|)\right)= \\
=T_{M}\left(R_{1}(x, z), \ldots, R_{n}(x, z)\right)=\tilde{R}_{A, T}(x, z) .
\end{gathered}
$$

Consider $x \leq y$ and $y>z$. If $x \leq z$ then the inequality $T^{*}\left(\tilde{R}_{A, T}(x, y), \tilde{R}_{A, T}(y, z)\right) \leq \tilde{R}_{A, T}(x, z)$ holds since $\tilde{R}_{A, T}(x, z)=1$. Therefore we observe the case when $x>z$. Thus $z<x \leq y$ and 


$$
\begin{gathered}
T^{*}\left(\tilde{R}_{A, T}(x, y), \tilde{R}_{A, T}(y, z)\right)= \\
=\tilde{R}_{A, T}(y, z)=T_{M}\left(R_{1}(y, z), \ldots, R_{n}(y, z)\right)= \\
=T_{M}\left(g_{1}(|y-z|), g_{2}(|y-z|), \ldots, g_{n}(|y-z|)\right) \leq \\
\leq T_{M}\left(g_{1}(|x-z|), g_{2}(|x-z|), \ldots, g_{n}(|x-z|)\right)= \\
=T_{M}\left(R_{1}(x, z), \ldots, R_{n}(x, z)\right)=\tilde{R}_{A, T}(x, z) .
\end{gathered}
$$

Consider $x>y$ and $y>z$, then $x>z$. Therefore

$$
\begin{gathered}
T^{*}\left(\tilde{R}_{A, T}(x, y), \tilde{R}_{A, T}(y, z)\right)= \\
=T^{*}\left(T_{M}\left(R_{1}(x, y), \ldots, R_{n}(x, y)\right)\right. \\
\left.T_{M}\left(R_{1}(y, z), \ldots, R_{n}(y, z)\right)\right) \leq \\
T_{M}\left(T^{*}\left(R_{1}(x, y), R_{1}(y, z)\right), T^{*}\left(R_{1}(x, y), R_{2}(y, z)\right), \ldots,\right. \\
\ldots, T^{*}\left(R_{n}(x, y), R_{n}(y, z)\right) \leq \\
\left.T_{M}\left(T^{*}\left(R_{1}(x, y), R_{1}(y, z)\right), R_{2}(x, y)\right), R_{2}(y, z)\right), \ldots \\
\ldots, T^{*}\left(R_{n}(x, y), R_{n}(y, z)\right) \leq \\
\leq T_{M}\left(R_{1}(x, z), \ldots, R_{n}(x, z)\right)=\tilde{R}_{A, T}(x, z)
\end{gathered}
$$

By the last we have proven the following:

Conclusion 2. If $R_{i}$ for all $i \in\{1, \ldots, n\}$ are $T^{*}$-transitive fuzzy relations given by formula 2, $T=T_{M}, A$ is an additive aggregation function then $\tilde{R}_{A, T_{M}}$ is also a $T^{*}$-transitive fuzzy relation.

We finish by the following remark:

Remark 3. If all $R_{i}, i \in\{1, \ldots, n\}$ are $T^{*}$-transitive, $T\left(R_{1}\left(x_{1}, y_{1}\right), \ldots, R_{n}\left(x_{n}, y_{n}\right)\right)=$ $=T\left(R_{1}\left(x_{1}^{\prime}, y_{1}^{\prime}\right), \ldots, R_{n}\left(x_{n}^{\prime}, y_{n}^{\prime}\right)\right)$ if

$A\left(x_{1}, \ldots, x_{n}\right)=A\left(x_{1}^{\prime}, \ldots, x_{n}^{\prime}\right)$ and

$A\left(y_{1}, \ldots, y_{n}\right)=A\left(y_{1}^{\prime}, \ldots, y_{n}^{\prime}\right)$ and $T$ dominates $T^{*}$ then $\tilde{R}_{A, T}$ is also $T^{*}$-transitive. The proof is obvious but this case reduces to pointwise aggregation and the aggregation of vectors does not play role.

\section{Conclusion}

The concept of aggregation of binary fuzzy relations studied in this paper is important in many practical applications. Studying conditions necessary for preservation of $T$-transitivity considered in this paper we are covering only some special cases. In the future studies we want to determine more general cases when $T$-transitivity holds for aggregated fuzzy relation.

Also it is important to know whether some particular properties of binary fuzzy relations, e.g. reflexivity, symmetry, associativity etc, are preserved in the aggregated object. Knowing this we are able to speak about aggregated order, equivalence and other special types of relations. This is another direction for the study initiated in this paper.

\section{References}

[1] B. De Baets and R. Mesiar, Pseudo-metrics and T-equivalences, Journal of Fuzzy Mathematics, 5:471-481, 1997.
[2] U. Bodenhofer, A similarity-based generalization of fuzzy orderings preserving the classical axioms, Internat. J. Uncertain. Fuzziness Knowledge-Based Systems, 8(5):593-610, 2000.

[3] U. Bodenhofer, Representations and constructions of similarity-based fuzzy orderings, Fuzzy Sets and Systems, 137:113-136, 2003.

[4] U. Bodenhofer, B. De Baets and J. Fodor, A compendium of fuzzy weak orders: representations and constructions, Fuzzy Sets and Systems, 158:593-610, 2007.

[5] U. Bodenhofer and J. Küng, Fuzzy orderings in flexible query answering systems, Soft Computing, 8(7):512-522, 2004.

[6] J. Fodor and M. Roubens, Fuzzy Preference Modelling and Multicriteria Decision Support, Kluwer Academic Publishers, Dordrecht, 1994.

[7] M. Grabisch, J.-L. Marichal, R. Mesiar and E. Pap, Aggregation Functions (Encyclopedia of Mathematics and its Applications), Cambridge University Press, UK, 2009.

[8] U. Höhle, N. Blanchard, Partial ordering in L-underdeterminate sets, Inf. Sci., 35:133-144, 1985.

[9] E.P. Klement, R. Mesiar and E. Pap, Triangular Norms, Kluwer Academic Publishers, The Netherlands, 2002.

[10] R. Mesiar, T. Calvo and G. Mayor, Aggregation Operators: New Trends and Applications (Studies in Fuzziness and Soft Computing), PhysicaVerlag, Heidelberg, New York, 2002.

[11] H.T. Nguyen, A note on the extension principle for fuzzy sets, Journal of Mathematical Analysis and Applications, 64:369-380, 1978.

[12] S.Saminger, R.Mesiar, U.Bodenhofer, Domination of aggregation operators and preservation of transitivity, International Journal of Uncertainty, Fuzziness and Knowledge-Based Systems, 10(Suppl.):11-35, 2002.

[13] L.A. Zadeh, Similarity relations and fuzzy orderings, Inform. Sci., 3:177-200, 1971.

[14] L.A. Zadeh, The concept of a linguistic variable and its applications in aproximate reasoning, Inform. Sci., 8:199-249; 301-357, 1975; 9:43-80, 1975. 